\title{
Temporal Bone and Vertebral Metastases of Papillary Thyroid Carcinoma Manifesting as Cranial Nerve VII Palsy and Spinal Cord Compression
}

\author{
Mark Anthony Sandoval and Laura Rosario T. Acampado \\ Section of Endocrinology, Diabetes and Metabolism, Department of Medicine, \\ College of Medicine and Philippine General Hospital \\ University of the Philippines Manila
}

\begin{abstract}
Cranial nerve VII palsy and spinal cord compression due to bone metastases are unusual manifestations of metastatic papillary thyroid carcinoma. Presented here is the case of a 54-year old man, known to have a goiter, but seeking consult only after he developed right facial weakness. Work up revealed a mass causing lysis of the right temporal bone with resultant facial nerve involvement. The temporal mass was eventually proven to be metastatic papillary carcinoma. The thyroid was subsequently removed, confirming that it was the primary location of papillary carcinoma, follicular variant. Two weeks after total thyroidectomy, he eventually developed symptoms of spinal cord compression due to vertebral metastases. He received external beam radiation therapy to the spine, radioactive iodine and levothyroxine for TSH suppression. The patient is alive 3 years after the diagnosis but the disease has left him with several disabilities.
\end{abstract}

Keywords: thyroid cancer, papillary thyroid carcinoma, bone metastasis, vertebral, facial nerve (CN VII) palsy, spinal cord compression

\section{The Case}

A 54-year-old male consulted for right-sided facial paralysis. Aside from a thyroid mass that was present for the past 6 years, he had no other known illnesses. No previous consults were made for the thyroid mass. He did not have seizures, loss of consciousness, behavioral changes, severe headache, vomiting, gait disturbance, deafness, tinnitus, otalgia nor otorrhea. He denied palpitations, weight loss, tremors, heat intolerance, hoarseness, dysphagia and dyspnea.

Physical examination revealed heart rate of 68 per minute and blood pressure of $120 / 90 \mathrm{mmHg}$. Very notable was peripheral or lower motor neuron facial weakness on the right. Extra-ocular muscles were intact. There were no sensory deficits on the face. There was no gross hearing defect. The rest of the cranial nerve examination was normal. Motor and sensory examination of the extremities was likewise normal. There was no dysmetria, dysdiadochokinesia nor ataxia. There was a $4 \times 3 \mathrm{~cm}$ hard mass on the right thyroid lobe. No neck nodes were palpated.

Initial assessment was Bell's palsy and a nontoxic solitary thyroid nodule.

\section{Diagnostic Work-up}

A CT scan of the head with emphasis on the temporal bone revealed an avidly enhancing, calcified mass

ISSN 0857-1074

Copyright (C) 2011 by the JAFES

Received January 24, 2011. Accepted March 26, 2011 measuring $4.0 \times 4.0 \times 4.8 \mathrm{~cm}$ that caused lysis of the right temporal bone. It obliterated much of the mastoid air cells, but spared the cochlea and semicircular canals. It had an intracranial extraaxial extension, occupying the right posterior fossa and compressing the right cerebellar hemisphere. It also extended inferiorly up to the level of the C2 vertebra (Figures 1, 2 and 3). Free thyroxine and thyrotropin levels were normal (Free T4 15.9 pmol/L and TSH $0.93 \mathrm{uIU} / \mathrm{mL}$ ).

A CT scan of the neck showed a $4.0 \times 4.3 \times 3.4 \mathrm{~cm}$ heterogeneously enhancing, predominantly solid mass with coarse calcifications occupying the right thyroid lobe. This caused displacement of the trachea to the left. There were no enlarged cervical lymph nodes seen (Figure 4).

Fine needle aspiration of the thyroid nodule was unsatisfactory as it was a bloody smear. Chest x-ray did not reveal any lung masses or bone lytic lesions.

\section{Clinical Course}

An incision biopsy of the right skull base mass was performed using the post-auricular approach. Microscopic examination revealed metastatic papillary carcinoma (Figure 5). 


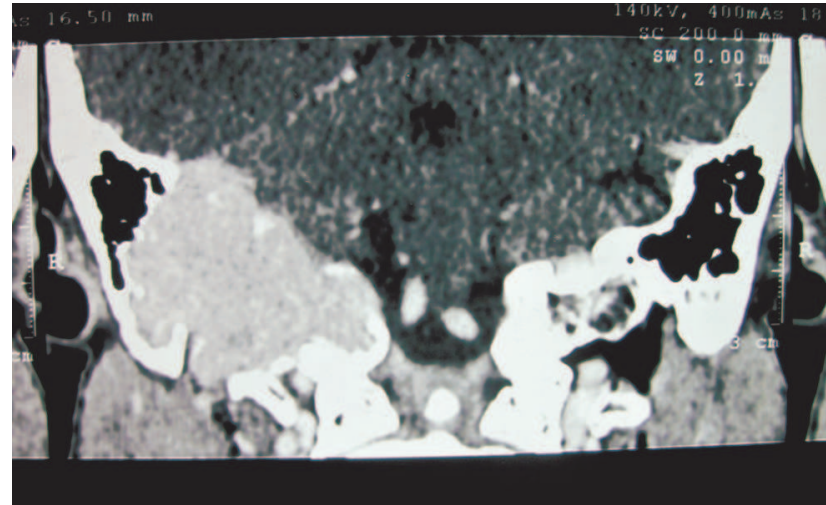

Figure 1. CT scan (coronal view) showing an enhancing mass with calcification at the right temporal bone with involvement of the mastoid air cells.

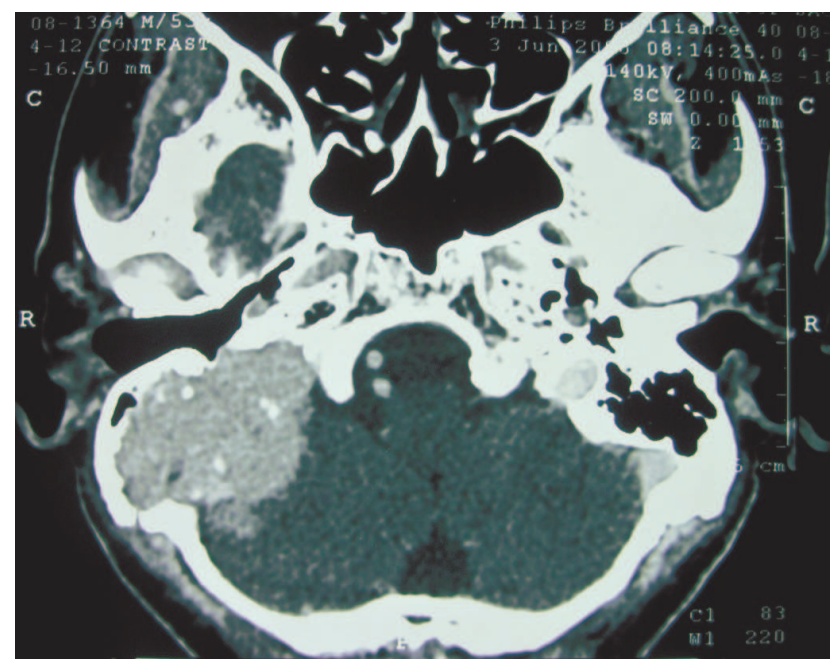

Figure 2. CT scan (axial view) showing a right temporal bone mass with extraaxial extension into the right posterior fossa, compressing the right cerebellar hemisphere.

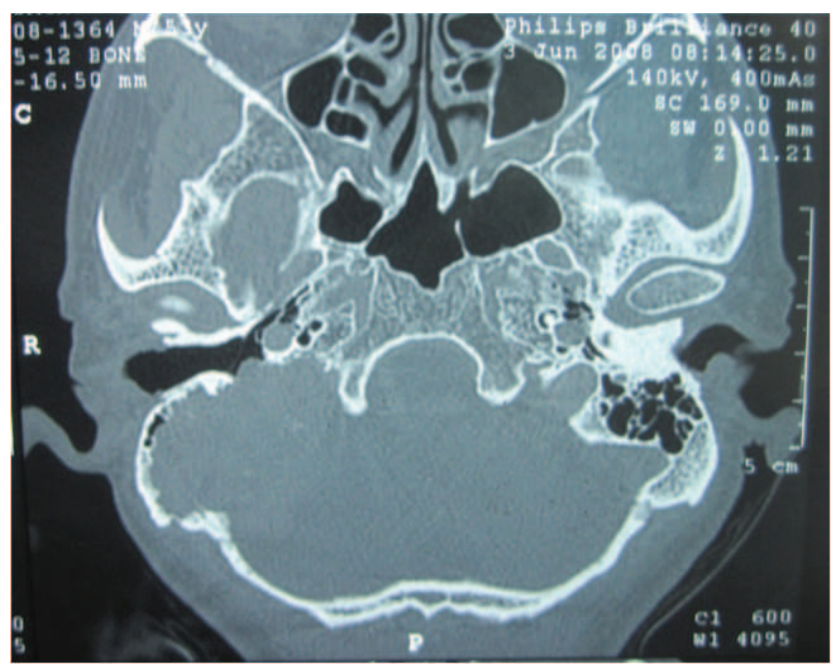

Figure 3. CT scan (axial view) bone window showing lysis of the right temporal bone with disappearance of most of the mastoid air cells.

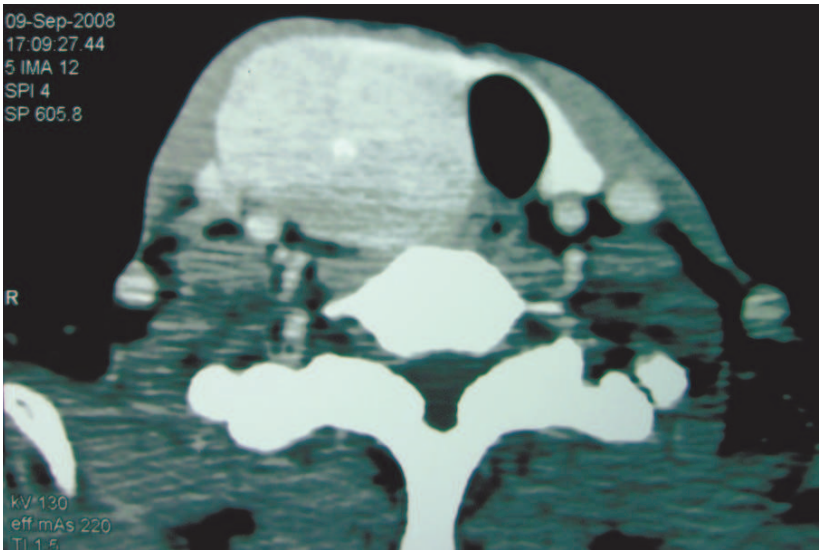

Figure 4. CT scan of the neck (axial view) showing a mass with calcification occupying the right thyroid lobe. Note the displacement of the trachea to the left.

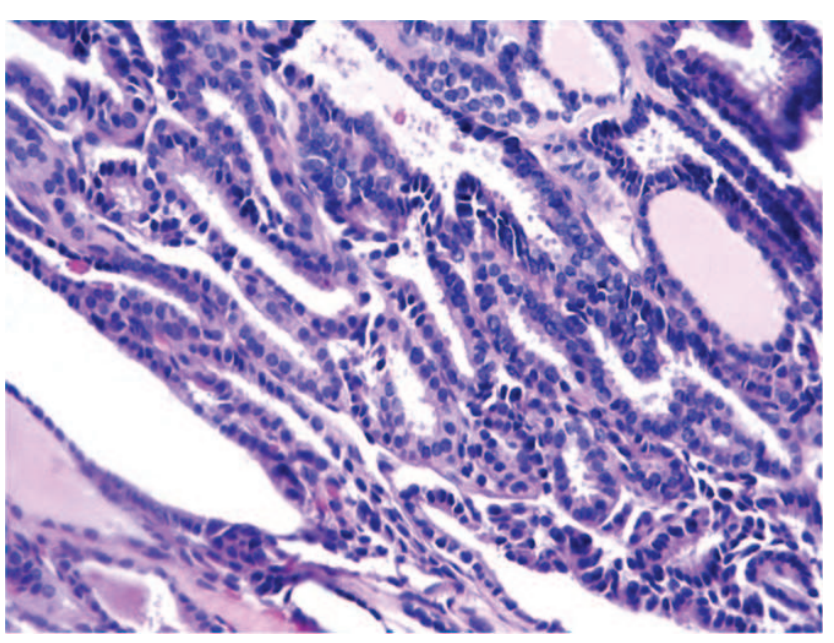

Figure 5. Microscopic examination of the incision biopsy of the right temporal bone mass showing metastatic papillary thyroid carcinoma (400x magnification, H\&E stain).

The patient first underwent craniotomy, excision of tumor and partial mastoidectomy on the right. Histopathologic examination revealed metastatic carcinoma, favoring a thyroid origin. Thirteen days after the craniotomy, total thyroidectomy was performed. Histopathologic examination revealed papillary thyroid carcinoma, follicular variant, with involvement of the right recurrent laryngeal nerve. Post-operative staging was T4N0M1 (Stage IV).

Within two weeks after thyroidectomy while waiting for some time to allow TSH to increase in preparation for radioactive iodine therapy, he developed back pain, bilateral leg weakness (motor strength 2/5), decreased sensation on both lower extremities and urinary retention.

Magnetic resonance imaging of the spine revealed multiple masses causing destruction of the T6, L1 and L4 vertebrae, with compression of the spinal cord at the T5-T6 
level. Aside from these vertebral masses, abnormal signals were also seen at the T8, T9 and T12 vertebrae.

For the spinal cord compression, he received 30 Gy of external beam radiation directed at the thoracic spine. This resulted in improved sensory function only. Patient remained paraplegic and wheelchair-bound. Urinary retention required the insertion of an indwelling urethral catheter.

Three months after thyroidectomy, he returned for radioactive iodine-131 therapy given at a dose of $200 \mathrm{mCi}$ (7400 MBq). He was pre-treated with oral dexamethasone $5 \mathrm{mg}$ three times daily to prevent worsening of spinal cord compression.

Post-treatment whole body iodine-131 scan revealed functioning thyroid tissue remnants in the neck. There was tracer uptake at the midline thorax, compatible with the known metastases seen on spinal MRI. No tracer uptake was seen at the cranium suggesting that excision of the temporal bone metastasis was complete or that the mass was poor in taking up the radioisotope.

Three months after radioactive iodine, motor strength of the lower extremities improved to $4 / 5$ but patient remained non-ambulatory. Urinary retention was resolved as he no longer required a urethral catheter. Thyroglobulin level remained elevated at $310.4 \mathrm{ng} / \mathrm{mL}$ at a TSH level of $0.7 \mathrm{uIU} / \mathrm{mL}$, while on levothyroxine 125 $\mathrm{mcg} /$ day, suggestive of persistent disease.

The patient was advised another dose of radioactive iodine therapy and possible spine surgery but declined. He opted for palliative treatment with opioid analgesics and continued levothyroxine treatment for $\mathrm{TSH}$ suppression (increased to $150 \mathrm{mcg} /$ day). The patient went back to their home province which is $500 \mathrm{~km}$ away from our medical center. The patient is alive three years after the diagnosis but he has not returned for follow-up since.

Final diagnosis was papillary thyroid carcinoma, follicular variant, T4N0M1 (Stage IV) with local invasion to the right recurrent laryngeal nerve (T4) and metastasis (M1) to the following: right temporal bone with involvement of the right facial (CN VII) nerve and with intracranial extraaxial extension to the right posterior fossa, and thoracolumbar vertebrae causing spinal cord compression.

\section{Discussion}

Well-differentiated thyroid carcinomas, under which the histologic types papillary and follicular belong, are associated with a good prognosis and impressive causespecific long-term survival. However, they can also be metastatic, with the lungs and bone being the most common sites of distant spread. ${ }^{1}$
Follicular carcinoma is generally known to be associated more with bone metastases than papillary carcinoma. In a retrospective study involving metastatic differentiated thyroid cancer, follicular carcinoma was found to be the primary tumor for bone metastases in $71 \%$ of cases, while papillary carcinoma accounted for only $17 \% .^{2}$ Also, the bone was the sole site of metastasis in only $12 \%$ of papillary carcinoma but in $36 \%$ of follicular carcinomas. ${ }^{3}$

In a study of temporal bone metastases, there was only a single case of thyroid cancer out of 47 metastases involving 76 temporal bones. This turned out to be follicular carcinoma. The most common tumor spreading to the temporal bone is breast cancer. ${ }^{4}$

In a case report from India, temporal bone metastasis from differentiated thyroid carcinoma was presented, but again this one proved to be a follicular carcinoma rather than a papillary carcinoma. ${ }^{5}$ Medullary thyroid carcinoma has been reported as well to involve the temporal bone. ${ }^{6}$ As of this writing, the author's literature search did not reveal any other case of temporal bone metastasis due to papillary thyroid carcinoma.

Thus, the clinical behavior exhibited by this tumor is uncommon for a papillary thyroid carcinoma but would have been expected of a follicular thyroid carcinoma.

For the case being presented here, craniotomy with excision of the temporal bone mass was done first prior to thyroidectomy. The rationale was to avoid the theoretical risk of increased intracranial pressure and herniation that might result from enlargement of the mass once thyroid stimulating hormone (TSH) increases as a consequence of thyroidectomy. Since the mass extended into the posterior cranial fossa, a small increase in tumor size may result in catastrophic tentorial herniation because of the confined space in this region of the cranium.

This phenomenon might actually explain why the patient developed spinal cord compression after he underwent total thyroidectomy. There might have been vertebral metastases to begin with but these were not causing symptoms initially. It is plausible that the increase in TSH that followed thyroidectomy caused the vertebral metastases to enlarge and cause spinal cord compression.

The rise in TSH after thyroidectomy is actually awaited before radioactive iodine therapy is given since this would facilitate uptake of the ablative radioisotope by the tumor cells. The recommended value of $25-30 \mathrm{uIU} / \mathrm{mL}$ is usually achieved within a month after total thyroidectomy. One option to avoid having to wait for this period of time to elapse is to administer recombinant human TSH (rhTSH) two days before giving radioactive iodine therapy. Recombinant human TSH, however, is not readily available in our local setting and the cost is prohibitive. 
The patient was not able to follow-up anymore after he declined a second dose of radioactive iodine and possible spine surgery. He just opted for palliative treatment. The patient is alive three years after the diagnosis but the disease has left him with significant debilities: facial weakness from the temporal bone metastasis, hoarseness from the recurrent laryngeal nerve involvement, and paraparesis from the vertebral metastases.

\section{Learning points}

- Cranial nerve VII palsy and spinal cord compression are unusual manifestations of papillary thyroid cancer.

- Facial weakness was due to facial nerve (CN VII) involvement from temporal bone metastasis.

- Weakness and decreased sensation of the lower extremities, and urinary retention were due to spinal cord compression from thoracolumbar vertebral metastases.

- Though follicular carcinoma is more commonly known to metastasize to bone, this case demonstrates that papillary carcinoma can also widely metastasize to bony structures (temporal bone and vertebrae) with resultant neurologic deficits and debilitating consequences.
- The authors' literature search did not reveal any other case of temporal bone metastases caused by papillary thyroid carcinoma.

\section{Acknowledgements}

We are thankful to the Department of Pathology of the University of the Philippines College of Medicine for the photomicrographs.

\section{References}

1. Schlumberger MJ. Papillary and follicular thyroid carcinoma. New England Journal of Medicine 1998; 338 (5): 297-306.

2. Bernier MO, Leenhardt L, Hoang C, Aurengo A et al. Survival and therapeutic modalities in patients with bone metastases of differentiated thyroid carcinomas. Journal of Clinical Endocrinology and Metabolism 2001; 86 (4): 1568-1573.

3. Durante C, Haddy N, Baudin E,Leboulleux S et al. Long-term outcome of 444 patients with distant metastases from papillary and follicular thyroid carcinoma: benefits and limits of radioiodine therapy. Journal of Clinical Endocrinology and Metabolism 2006; 91 (8): 2892-2899.

4. Gloria-Cruz TI, Schachern PA, paparella MM, Adams GL and Fulton $\mathrm{SE}$. Metastases to temporal bones from primary nonsystemic malignant neoplasms. Archives of Otorhinoloaryngology - Head and Neck Surgery 2000; 126: 209-214.

5. Raj A, Sayal A, Meher R, Girhotra M, Jain S. Temporal bone metastasis from thyroid. Indian Journal of Cancer 2010; 47 (2): 228229.

6. Redleaf MI, Bauman NM, Robinson RA, Gantz BJ. Medullary carcinoma of the thyroid metastatic to the temporal bone. Journal of Laryngology and Otology 1995; 109: 1200-3.

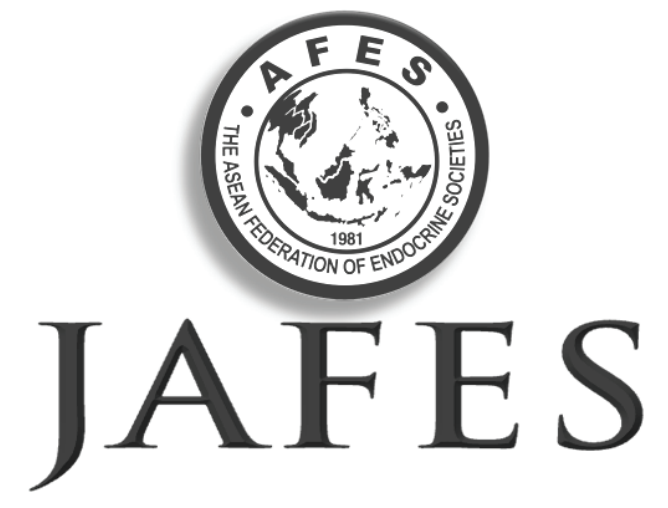

Experience the new JAFES. Visit us at www.ASEAN-endocrinejournal.org. 